Check for updates

Cite this: Org. Biomol. Chem., 2022, 20, 5999

Received 10th January 2022

Accepted 4th February 2022

DOI: $10.1039 / \mathrm{d} 2 \mathrm{ob00066 \textrm {k }}$

rsc.li/obc

\title{
Supramolecular self-associating amphiphiles: determination of molecular self-association properties and calculation of critical micelle concentration using a high-throughput, optical density based methodology $\dagger$
}

\author{
Andzelika Rutkauskaite, ${ }^{a}$ Lisa J. White, ${ }^{a}$ Kira L. F. Hilton, ${ }^{a}$ Giacomo Picci, ${ }^{b}$ \\ Lorraine Croucher, ${ }^{c}$ Claudia Caltagirone (D)*b and Jennifer R. Hiscock (D) *a
}

\begin{abstract}
Supramolecular self-associating amphiphiles are a class of amphiphilic salt, the anionic component of which is 'frustrated' in nature, meaning multiple hydrogen bonding modes can be accessed simultaneously. Here we derive critical micelle concentration values for four supramolecular self-associating amphiphiles using the standard pendant drop approach and present a new high-throughput, optical density measurement based methodology, to enable the estimation of critical micelle concentrations over multiple temperatures. In addition, we characterise the low-level hydrogen bonded self-association events in the solid state, through single crystal X-ray diffraction, and in polar organic DMSO- $d_{6}$ solutions using a combination of ${ }^{1} \mathrm{H}$ NMR techniques. Moving into aqueous ethanol solutions $\left(\mathrm{EtOH} / \mathrm{H}_{2} \mathrm{O}\right.$ or $\mathrm{EtOH} /$ $\left.\mathrm{D}_{2} \mathrm{O}(1: 19 \mathrm{v} / \mathrm{v})\right)$, we also show these amphiphilic compounds to form higher-order self-associated species through a combination of ${ }^{1} \mathrm{H}$ NMR, dynamic light scattering and zeta potential studies.
\end{abstract}

\section{Introduction}

Amphiphilic molecules have the capability to naturally selfassociate into a variety of structures, including but not limited to vesicles, bilayers, nanotubes and micelles. ${ }^{1,2}$ The resultant self-associated structures formed are stabilised through an assortment of intermolecular non-covalent interactions such as: hydrogen bonding; ion-ion interactions; van der Waals forces; $\pi-\pi$ stacking; and hydrophobic/hydrophilic interactions. $^{3-5}$ The importance of this class of compounds cannot be underestimated, with examples utilised as antimicrobial agents, ${ }^{6-8}$ anticancer agents, ${ }^{9-11}$ drug delivery vehicles, ${ }^{12-14}$ and within the oil related industries. ${ }^{15,16}$

\footnotetext{
${ }^{a}$ School of Chemistry and Forensics, University of Kent, Canterbury, Kent, CT2 7NH, UK. E-mail: J.R.Hiscock@Kent.ac.uk

${ }^{b}$ Dipartimento di Scienze Chimiche e Geologiche, Università degli Studi di Cagliari, SS 554 Bivio per Sestu, 09042 Monserrato, CA, Italy. E-mail: ccaltagirone@unica.it ${ }^{c} B M G$ Labtech, 8 Bell Business Park, Aylesbury, Bucks, HP19 8JR England, UK $\dagger$ Electronic supplementary information (ESI) available: This includes experimental methodologies, molecular characterisation data, single crystal X-ray data, high resolution mass spectrometry data, NMR, zeta potential data, dynamic light scattering data, tensiometry data and plate reader data. CCDC 1999015. For ESI and crystallographic data in CIF or other electronic format see DOI: 10.1039/ d2ob00066k
}

Amphiphiles, in aqueous solutions, have two simultaneous tendencies: to aggregate within the solvent bulk to form structures such as micelles, or to adsorb at the interface, ${ }^{17}$ a process which affects surface tension. ${ }^{18}$ When designing amphiphilic systems, a key characterisation parameter for both fundamental study and commercial development is the critical micelle concentration (CMC). CMC is commonly defined as the concentration at which the solution interface becomes saturated with amphiphilic molecules. ${ }^{19}$ After this point any further increase in amphiphile concentration will result in the formation of higher-order self-associated species within the bulk of the solution.

Many different methods have been developed to enable CMC determination, through the monitoring of physical properties, such as electrical conductivity, surface tension, density, dynamic light scattering (DLS), refractive index, fluorescence emission, and UV-visible absorption with respect to amphiphile concentration. ${ }^{19-28}$ However, these techniques all exhibit major drawbacks such as high costs, need for specialist equipment, large sample volumes, ineffective environmental control as well as low throughput methodology, and contamination issues that could lead to some inaccuracy in CMC determination. For example, commonly employed DLS methodology suffers from a lack of automation and comparatively large sample volumes $(\approx 1 \mathrm{~mL}){ }^{28}$ In addition, the amphiphilic 
species present at a solution interface may also exist in dynamic equilibrium with larger aggregated structures within the solutions bulk at concentrations below the CMC, leading to the reporting of inaccurate results. ${ }^{29-32}$

Given these limitations, CMC is more commonly reported as the point at which surface tension of a aqueous solution no longer decreases with increasing amphiphile concentration. ${ }^{33}$ There are a number of experimental procedures available to measure surface tension including the (i) Wilhemly plate method. ${ }^{34}$ However, this method often suffers from contamination issues and requires comparatively large sample volumes; while the (ii) Pendant drop method ${ }^{20,35}$ offers little control over environmental parameters such as temperature, the variation of which is known to affect the CMC. ${ }^{36,37}$

A number of fluorescent probes have also been developed to enable CMC determination. ${ }^{22,24}$ However, this methodology requires the addition of molecular dyes/probes to a system, ${ }^{38}$ which have the capability to influence the self-association events present in solution, and as a result the CMC. ${ }^{39}$

Recent work by Cai and co-workers has gone some way towards removing those limitations commonly associated with CMC determination such as low throughput, need for specialised equipment or chemical probes, large sample volumes and ineffective environmental control, through the use of microplate reader technology. ${ }^{40}$ This readily available biological equipment can be fully automated, environmentally controlled and monitor up to 384 samples simultaneously. Here the authors monitor the comparative change in surface curvature (and thus change in surface tension) of an amphiphile containing solution within the wells of a microwell plate. The change in surface curvature was found to cause substantial signal differences in spectroscopic readings, recorded using a vertical detecting light beam. However, this methodology does suffer from limitations associated with highly curved liquid surfaces such as water, where measurement sensitivity is decreased.

Supramolecular self-associating amphiphiles (SSAs) are a class of 'frustrated' amphiphilic salts, the anionic component of which contains an uneven number of covalently linked hydrogen bond donating and accepting moieties, meaning that this amphiphilic unit can access multiple hydrogen bonding modes simultaneously. ${ }^{31,32,41-44}$ This class of compound has been shown to act as novel antimicrobial agents, ${ }^{45-47}$ therapeutic enhancement agents, ${ }^{48,49}$ as electrochemical agents within flow cell technologies ${ }^{50}$ and potential drug delivery vehicles. ${ }^{51}$ Vital to developing SSAs towards use within the clinic is the derivation of CMC within an aqueous environment. However, due to the large compound libraries often required to identify a lead therapeutic agent, alongside current experimental limitations associated with the aforementioned CMC derivation techniques, we have identified the need for a step-change in CMC derivation methodologies. In response to this need, we present a novel plate reader-based methodology, to enable the automated, high-throughput collection of optical density (OD) data from which we derive the CMC for four SSAs (Fig. 1) as a proof of principle, in an EtOH/

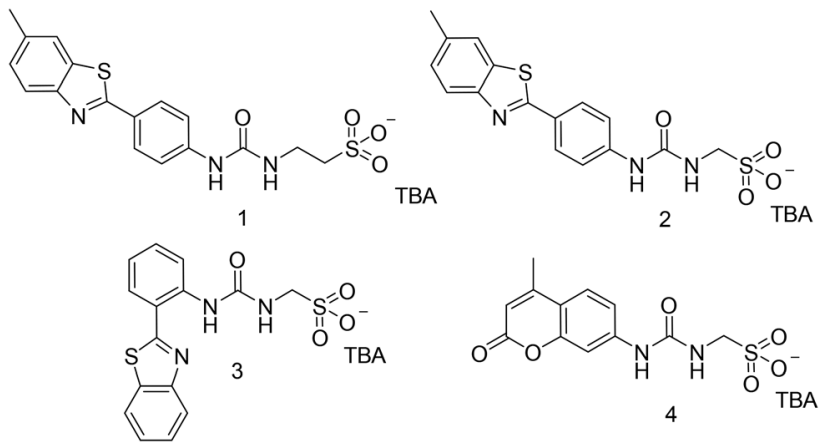

Fig. 1 Chemical structure of SSAs 1-4. TBA = tetrabutylammonium.

$\mathrm{H}_{2} \mathrm{O}(1: 19 \mathrm{v} / \mathrm{v})$ solution under environmentally controlled conditions.

\section{Results and discussion}

Compound 1 was synthesised through the reaction of the appropriate benzothiazolyl aniline with carbonyl diimidazole (CDI) and tetrabutylammonium (TBA) aminoethanesulfonate in chloroform, affording the pure product as a pale yellow solid in a yield of $61 \%$. Compound 4 was synthesised through the reaction of the appropriate amino coumarin with triphosgene and TBA aminomethanesulfonate in ethyl acetate, affording the pure product as a white solid in a yield of $45 \%$. Compounds 2 and 3 were synthesised through previously published methods. ${ }^{31}$ See experimental for synthetic details. $\dagger$

\section{Amphiphile self-association}

Initially, physicochemical analysis of 1-4 was performed to enable characterisation of both molecular low-level and aggregate high-level self-association events observed for this class of compound. ${ }^{51}$ As illustrated in Fig. 2, single crystal X-ray diffraction studies showed the anionic component of $\mathbf{4}$ to dimerise in

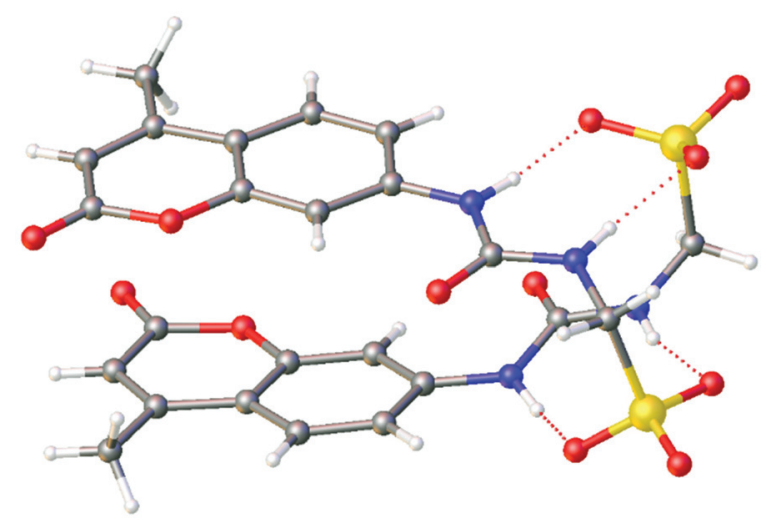

Fig. 2 Single crystal $X$-ray structure of 4 . The TBA counter cations have been omitted for clarity. Grey = carbon; white = hydrogen; blue = nitrogen; yellow = sulphur; red = oxygen. Red dashed line indicates hydrogen bonding. $C C D C=1999015$. $\dagger$ 
Table 1 Summary of the results collected for the anionic component of SSAs 1-4 from: quantitative ${ }^{1} \mathrm{H}$ NMR studies conducted in (i) DMSO- $\mathrm{d}_{6}$, standardised with $1.0 \% \mathrm{DCM}$ at $112 \mathrm{mM}$ and, (ii) $\mathrm{D}_{2} \mathrm{O}$ standardised with $5.0 \%$ ethanol at $5.56 \mathrm{mM}$. Values given represent the proportion of compound to become NMR silent; SSA anion dimerisation constants $\left(k_{\text {dim }}\right)$, calculated from ${ }^{1} \mathrm{H}$ NMR dilution studies conducted in a DMSO- $\mathrm{d}_{6} / 0.5 \% \mathrm{H}_{2} \mathrm{O}$ mixture at $298 \mathrm{~K}_{;}^{52}{ }^{1} \mathrm{H}$ NMR DOSY (DMSO- $\mathrm{d}_{6} / 0.5 \% \mathrm{H}_{2} \mathrm{O}$ ) studies conducted at $298 \mathrm{~K}$. All quantitative ${ }^{1} \mathrm{H}$ NMR experiments were conducted with a delay time $\left(d_{1}\right)$ of $60 \mathrm{~s}$ at $298 \mathrm{~K}$

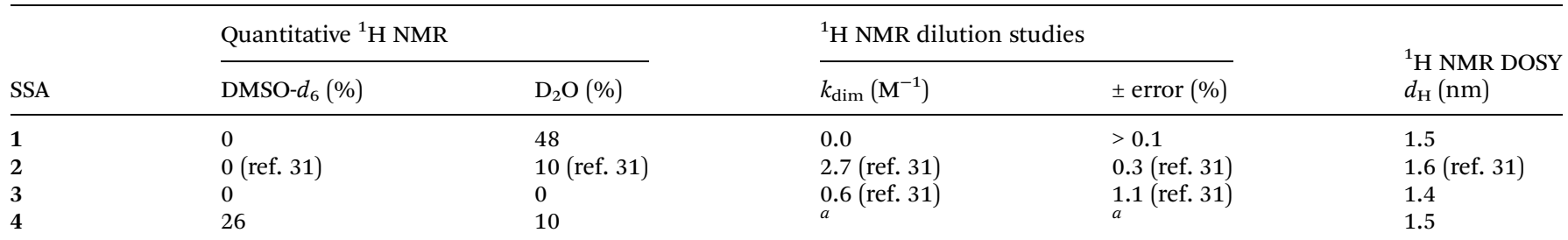

${ }^{a}$ Dimerisation constant could not be calculated due to the presence of higher-order self-association events. However, a downfield change in chemical shift with increasing concentration of SSA was observed, indicating the presence of self-associative intermolecular hydrogen bond formation.

the solid state. $\$$ These anionic dimers are stabilised through the formation of four hydrogen bonds, one from each $\mathrm{NH}$ hydrogen bond donating group to a different sulfonate oxygen atom (Fig. S22, and Table S5). $\dagger$

To verify the presence of hydrogen bonding events between the anionic units of 1-4 within the solution state, a combination of complimentary quantitative ${ }^{1} \mathrm{H}$ NMR studies (performed in DMSO- $d_{6}$ standardized with $1.0 \%$ DCM), ${ }^{1} \mathrm{H}$ NMR dilution studies (performed in DMSO- $d_{6} / 0.5 \% \mathrm{H}_{2} \mathrm{O}$ ) and ${ }^{1} \mathrm{H}$ NMR DOSY (in DMSO- $d_{6} / 0.5 \% \mathrm{H}_{2} \mathrm{O}$ ) studies were performed. The results of these studies have been summarised in Table 1.

Here quantitative ${ }^{1} \mathrm{H}$ NMR studies identify the presence and proportion of larger aggregated SSA species within the solution bulk. These larger aggregated species adopt solid-like properties and thus become NMR silent. ${ }^{51}$ Studies conducted in DMSO- $d_{6}$ standardized with 1\% DCM (Fig. S7-S12†) showed only the anionic component of SSA 4 , to undergo higher-order self-association processes under these experimental conditions. Here, $26 \%$ of the anionic component of 4 was observed to become NMR silent. We hypothesize this difference in aggregation behavior is due to the replacement of the comparatively hydrophobic benzothiazole (1-3) with the hydrophilic coumarin moiety (4).

To characterize the low-level SSA self-associated species present in a DMSO- $d_{6} / 0.5 \% \mathrm{H}_{2} \mathrm{O}$ solution, a combination of ${ }^{1} \mathrm{H}$ NMR DOSY and dilution studies were performed to enable the calculation of SSA hydrodynamic diameter $\left(d_{\mathrm{H}}\right)$ and strength of self-associative hydrogen bond formation respectively (Table 1). The results of ${ }^{1} \mathrm{H}$ NMR DOSY studies showed the anion and cation of 1-4 to diffuse at different rates, meaning the two ionic SSA components are not strongly coordinated under these experimental conditions. Further to this, the $d_{\mathrm{H}}$

\$A suitable crystal was obtained through slow evaporation of a EtOH/ $\mathrm{H}_{2} \mathrm{O}(1: 19$ $\mathrm{v} / \mathrm{v}$ ) solution containing the appropriate compound and the sample selected then mounted on a Rigaku Oxford Diffraction Supernova diffractometer. Data were collected using $\mathrm{Cu} \mathrm{K} \alpha$ radiation at $100 \mathrm{~K}$. The structure was solved with the ShelXS ${ }^{55}$ via Direct methods and refined with ShelXL ${ }^{56}$ on least squares minimisation. Olex $2^{57}$ was used as an interface to all ShelX programs. CCDC deposition number for the structure shown in Fig. $2=1999015 . \dagger$ calculated via the Stokes-Einstein equation from the appropriate average SSA diffusion constant, showed the anionic component of 1-4 to vary between $1.4-1.6 \mathrm{~nm}$, indicating the presence of lower-order dimeric species. In addition, for SSAs 1-3, the data generated from ${ }^{1} \mathrm{H}$ NMR dilution study data were found to fit dimerisation isotherms over more complex selfassociative isotherms,${ }^{52}$ providing complimentary evidence for the presence of anionic SSA hydrogen bonded dimeric species in solution.

When comparing the dimerisation constants calculated for 1-3, we had previously hypothesized that the decrease in dimerisation constant observed for $3\left(0.6 \mathrm{M}^{-1}\right)$ when compared to $2\left(2.7 \mathrm{M}^{-1}\right)$ was due to the presence of an intramolecular hydrogen bond (Fig. 3b), that prevented optimal intermolecular hydrogen bond formation, and as a result weakened any self-associative event. ${ }^{31}$ Interestingly, when analyzing those analogous data sets obtained with $\mathbf{1}$, we were unable to find any evidence of measurable SSA hydrogen bonded selfassociation. Here we again hypothesize that this may be due to intramolecular hydrogen bond formation within the anionic component of the SSA as shown in Fig. 3a. ${ }^{53}$

In an $\mathrm{EtOH} / \mathrm{D}_{2} \mathrm{O}(1: 19 \mathrm{v} / \mathrm{v})$ solution, quantitative ${ }^{1} \mathrm{H}$ NMR experiments confirm the presence of higher order self-associated species with solid-like properties for SSAs 1, 2 and 4 at $5.56 \mathrm{mM}$, see Table 1. Interestingly, unlike SSAs 1, 2 and 4, SSA 3 does not demonstrate any evidence of higher-order selfassociated structures under these experimental conditions, meaning that any larger aggregates that exist at these concen-

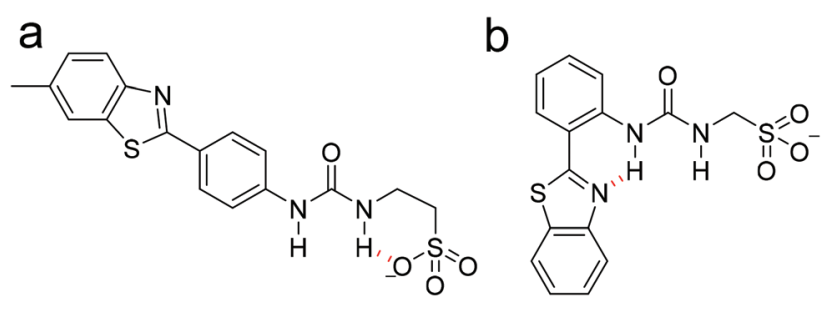

Fig. 3 Fypothesised intramolecular hydrogen bonds, shown in red, formed within the anionic component of (a) 1 and (b) 3 . 
trations either do not adopt any solid-like properties or exist at concentrations that are below the limit of detection for this methodology. We hypothesize that this is due to the presence of SSA intramolecular hydrogen bond formation events preventing effective SSA self-association, combined with effects relating to the positioning of hydrophobic/hydrophilic moieties within the SSA structure.

The $d_{\mathrm{H}}$ and stability of those higher-order self-associated structures produced by $\mathbf{1 - 4}$, in an $\mathrm{EtOH} / \mathrm{H}_{2} \mathrm{O}(1: 19 \mathrm{v} / \mathrm{v})$, were obtained via a combination of DLS and zeta potential measurements respectively (see Table 2). The $d_{\mathrm{H}}$ of those higher-order aggregates produced by $\mathbf{2 - 4}$ were found to maintain a similar size distribution, however the self-associated aggregates produced by 1 were found to have an average $d_{\mathrm{H}}>500 \mathrm{~nm}$, which may be due to the aggregation of smaller species or to the construction of larger singular structures.

Tensiometry experiments confirmed that SSAs 1-4 lower the surface tension of an EtOH/ $\mathrm{H}_{2} \mathrm{O}(1: 19 \mathrm{v} / \mathrm{v})$ solution, and thus exhibit surfactant properties. Therefore, a CMC was calculated for 1-4, using conventional pendant drop methodology. These values are reported in Table 2. This method was selected based on institutional equipment availability, the need for small sample sizes to conserve material and ease of use. Of these four SSAs, 2 was found to exhibit the lowest CMC at $0.50 \mathrm{mM}$, with the suspected presence of competitive intramolecular hydrogen bonding events within the anionic component of $\mathbf{1}$ and 3, hypothesised to contribute to an increase in CMC value to $1.92 \mathrm{mM}$ and $9.54 \mathrm{mM}$ respectively. However, the most dramatic increase in CMC was observed with the coumarin substituted SSA 4. Here, the presence of this comparatively hydrophilic residue is thought to cause the CMC to increase still further to $16.20 \mathrm{mM}$. However, when comparing the stability of those aggregates produced, decreasing zeta potential values (increasing aggregate stability) were found to correlate with decreasing CMC. Therefore, we also hypothesise that the stability of the higher-order aggregate produced is dependent on the hydrophilic nature of the aromatic substituent present within the anionic component of the SSA.

\section{Determination of critical micelle concentration via optical density (OD) well scan measurements}

Although CMC determination was possible for SSAs 1-4 using the pendant drop method (Table 2), we were unable to elucidate the CMC for this class of potential therapeutic agents at near physiological temperatures or establish the effect of temperature on the SSA CMC value. To overcome this limitation, we designed a CMC determination assay that enables environmental control of the sample to be studied and this parameter to be calculated through the collection of a series of optical density (OD) measurements.

OD microplate well scans have been shown as a powerful tool when assigning/understanding supramolecular selfassociation events. ${ }^{54}$ Here, we demonstrate how the data from these scans maybe processed to enable the calculation of CMC values with environmental control, in a high-throughput and automated manner, using comparatively low sample volumes and a standard 96-well microplate. These microplate well scans are able to record an OD reading for up to 177 different sections of a single well within a standard 96 well plate. Through analysis of these data, we show that it is possible to derive CMC values for amphiphiles 1-4. Here the equipment that we are using has a focal point of $0.3 \mathrm{~mm}$ so, in a 96 microwell plate with a well diameter of $6 \mathrm{~mm}$ there will be minimal overlap of these OD measurements. Where this minimal overlap occurs, the multipoint averaging approach used, enables this overlap to become an advantage, enabling us to confirm with certainty the trends that we are seeing. In addition, as we are using an absorbance assay there is unlikely to be light bleed through or photobleaching (as seen in luminescence or fluorescence) as adsorption is a quantitative measure and is therefore unaffected by the surrounding sample points. OD measurements were performed in the region 360-380 $\mathrm{nm}$ in which the four SSAs showed an absorption (Table 3). It should also be noted that it is not a requirement of this methodology to use the adsorption peak maxima, merely that the aggregated compound adsorbs at this wavelength.

A series of OD microplate well scans were obtained for solutions of 1-4 at various concentrations, temperatures and in

Table 3 OD wavelengths $(\mathrm{nm})$ used to obtain $\mathrm{CMC}$ values at $25-45^{\circ} \mathrm{C}$

\begin{tabular}{lllll}
\hline & SSA & & & \\
\cline { 2 - 5 } Wavelength & $\mathbf{1}$ & $\mathbf{2}$ & $\mathbf{3}$ & $\mathbf{4}$ \\
\hline & 370 & 360 & 385 & 386
\end{tabular}

Table 2 Summary of DLS, zeta potential and tensiometry data produced to characterise the higher-order self-associated aggregates of 1-4 formed in an EtOH/ $\mathrm{H}_{2} \mathrm{O}(1: 19 \mathrm{v} / \mathrm{v})$ solution. The $d_{\mathrm{H}}$ was calculated from DLS intensity distribution peak maxima data, obtained from a solution of the appropriate SSA $(5.56 \mathrm{mM})$ at $298 \mathrm{~K}$. The polydispersity index (PDI) for these DLS data is given in \%. Zeta potential measurements were also obtained at an SSA concentration of $5.56 \mathrm{mM}$ at $298 \mathrm{~K}$. Here the CMC was derived from surface tension measurements obtained at room temperature 20

\begin{tabular}{llllll}
\hline SSA & $d_{\mathrm{H}}(\mathrm{nm})$ & PDI $(\%)$ & Zeta potential $(\mathrm{mV})$ & CMC $(\mathrm{mM})$ & ${\text { Surface tension at CMC }\left(\mathrm{mN} \mathrm{m}{ }^{-1}\right)}$ \\
\hline $\mathbf{1}$ & 815 & 24.25 & -88 & 1.92 & 46.24 \\
$\mathbf{2}$ & $59,300($ ref. 31) & 27.21 & -101 (ref. 31) & 0.50 (ref. 31) & 46.50 (ref. 31) \\
$\mathbf{3}$ & $300($ ref. 31) & 26.71 & -79 (ref. 31) & 9.54 (ref. 31) & 48.71 (ref. 31) \\
$\mathbf{4}$ & 279 & 26.33 & -59 & 16.20 & 38.30
\end{tabular}

${ }^{a}$ Any higher-order self-associated aggregates produced at these concentrations exist are likely to exist as a minor component of this system. 

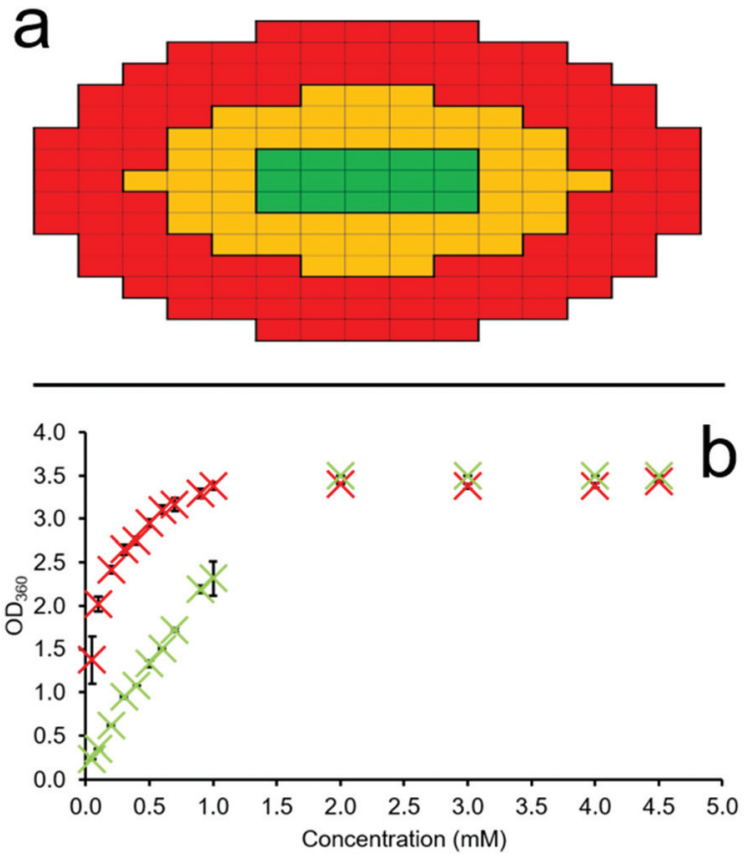

Fig. 4 (a) Diagram illustrating the 177 sections monitored during the OD well scan measurement process. Red $=110$ sections that are used to monitor the increased OD due to compound aggregation at the well interface. Orange $=52$ 'buffer zone' sections, not included in analysis. Green $=15$ control sections. (b) Example graph showing average $(n=3)$ $\mathrm{OD}_{360}$ values for the red and green sections for an $\mathrm{EtOH} / \mathrm{H}_{2} \mathrm{O}(1: 19 \mathrm{v} / \mathrm{v})$ solution of 2 obtained at $298 \mathrm{~K}$. Error - standard error of the mean.

triplicate, to ensure the validity of the data collected. Through comparison of average OD measurements obtained for different sections of each well (red and green - Fig. 4a) at different SSA concentrations we can estimate the CMC. This is made possible due to the propensity of an amphiphile to first aggregate at the sample interface (periphery of the well) before the sample bulk. Thus, through the monitoring of increasing amphiphile aggregation events at the well-solution interface (Fig. 4a - red), as indicated by increasing OD measurements, in comparison to those SSA aggregation events which occur within the central 'control' section of each well (Fig. 4a green), we may identify the concentration at which SSA aggregation events transition from the well interface to the central bulk, and thus define the CMC. If no SSA amphiphile aggregation events occur within the solution, then the OD measurements will remain uniform/proportional across the microwell plate as the concentration of the SSA is increased. It is the propensity of an amphiphile to form self-associated structures at the interface before initiating aggregate formation within the sample bulk that we are able to observe using this methodology.

The first step in this process is achieved through obtaining the multipoint average value for both the red 'interface' and green 'control' section of each microplate well independently. The multipoint averages obtained for each $n=3$ technical repeat are then further averaged and these data plotted to ensure statistical significance and reproducibility of those data obtained. An exemplar of these semi-processed data are illustrated in Fig. 4b. Here, we can clearly see the immediate increase in average OD values at the red 'interface' area of the well with increasing SSA concentration in comparison to the central green 'control' area. This is due to the primary amphiphile self-association events which occur at the sample interface over those species which exist in the central bulk of the solution at lower SSA concentrations.

Fig. $4 \mathrm{~b}$ also shows that we record OD readings of $\approx 3$ as the SSA concentration increases, corresponding to a sample containing a high proportion of solid-state aggregates, which in this case is what we want and expect to see. This methodology acknowledges the loss of a linear OD:SSA concentration relationship when recording such high OD values. However, it is because of this that we then take the ratio of these average $n$ $=3 \mathrm{OD}$ interface (red) and 'control' (green) values, using the average 'control' (green) OD values to standardize our data output and mitigate the impact of this limitation on the CMC values determined through the use of this methodology.

Through plotting the ratio of these average OD interface (red) and 'control' (green) values, it may become possible to identify three different concentration dependent phases within the higher-order SSA aggregation process. The first is the SSA concentration range in which the increasing ratio of average OD 'interface' (red) and 'control' area (green) values are directly proportional to the increase in SSA concentration, Fig. 5a-d - orange. This indicates that within this concentration range, the majority of the SSA in solution is aggregating at the well-solution interface. The second phase within this process is identified where the increasing concentration of SSA at the well-solution interface is no longer directly proportional to increasing SSA concentration. This means that the concentration of SSA higher-order aggregated species in the central 'control' region of the well is increasing by a larger amount then that at the well interface upon further SSA addition, Fig. $5 \mathrm{a}-\mathrm{d}-$ blue. The third phase exists where again the range in which the ratio of average OD red : green values are directly proportional to the increase in SSA concentration, Fig. 5a-d grey. However, in this instance this is because we now observe a directly proportional increase in SSA aggregation events at the 'interfacial' and 'central' regions of the well with increasing concentration of SSA, indicating that the CMC has been reached and exceeded.

To estimate CMC we then fit two linear trend lines to those data which fall within the first and third phase of the aggregation events observed (Fig. 5a-d - orange and grey) and report the CMC as the intersection of the linear trend lines associated with the first and third phases of SSA higher-order association events. The CMC values calculated for SSAs 1-4 at $298 \mathrm{~K}, 308 \mathrm{~K}$ and $318 \mathrm{~K}$ using this OD methodology are summarised in Table 4. Here, these data are provided alongside CMC values calculated using traditional pendant drop methodology as exemplified in Fig. 5e and $\mathrm{f}$.

A comparison of those CMC values obtained at room temperature (approximately $291 \mathrm{~K}-298 \mathrm{~K}$ ) via tensiometry, using the 

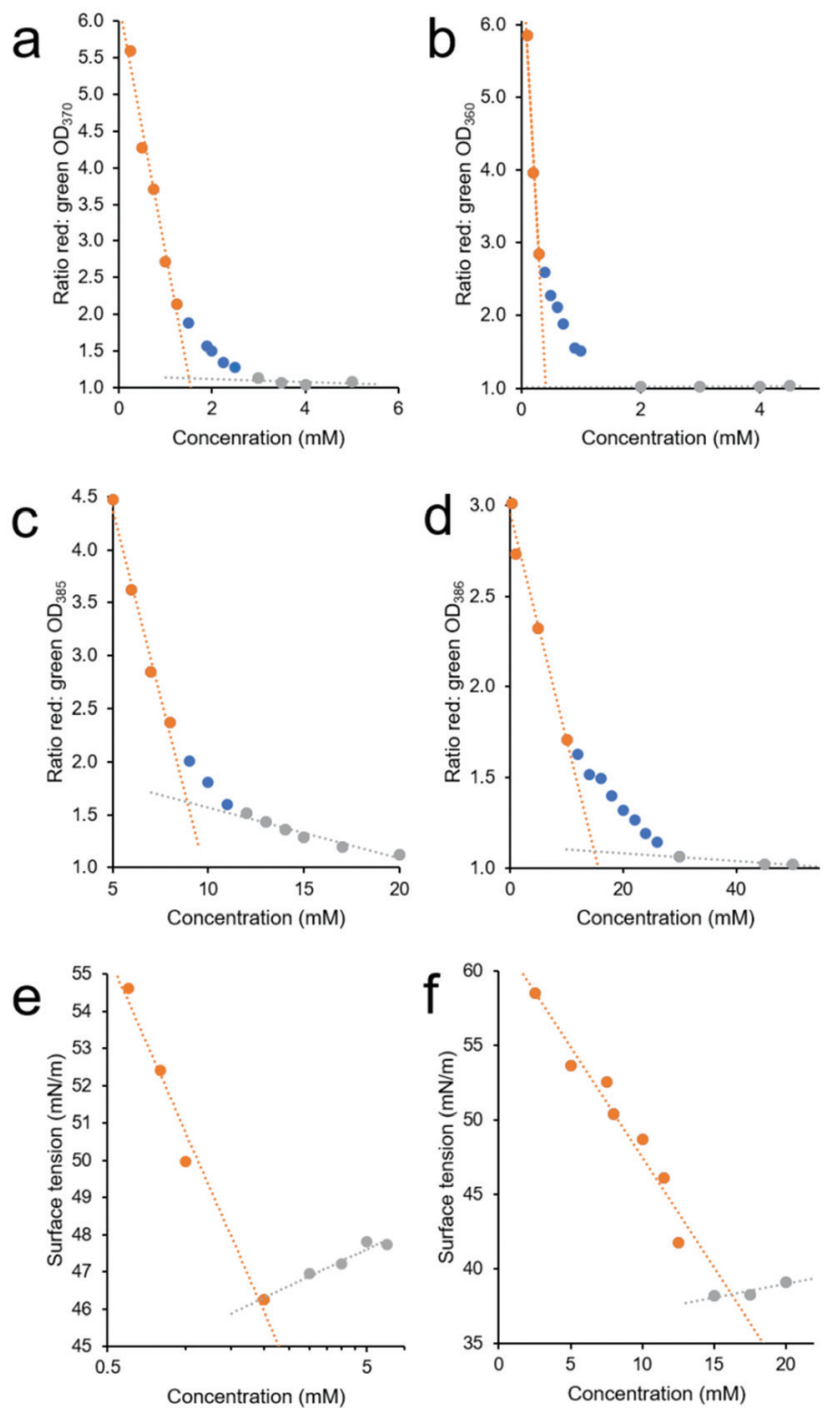

Fig. 5 (a-d) Graphs showing the ratio of average red:green section OD values (see Fig. $4 a)$, calculated from average $(n=3)$ OD well scan data obtained for 1 (4a), 2 (4b), 3 (4c), and 4 (4d) in an EtOH/ $\mathrm{H}_{2} \mathrm{O}(1: 19$ $\mathrm{v} / \mathrm{v}$ ) solution at $298 \mathrm{~K}$, with increasing compound concentration. (e and f) Graphs showing the change in surface tension, calculated from average $(n=3)$ tensiometer data obtained for $1(4 \mathrm{e})$ and $4(4 \mathrm{f})$ in an EtOH : $\mathrm{H}_{2} \mathrm{O} 1: 19$ solution at room temperature with increasing SSA concentration. In all cases the $\mathrm{CMC}$ value was defined as the point at which the orange and grey linear lines of best fit, fitted to the two linear portions of these data sets were found to intersect.

pendant drop method, and those obtained using our own OD based methodology showed the CMC values calculated using the pendant drop method to be generally higher than those calculated using the OD based methodology. The difference in CMC value calculated by these two different methods we believe may be explained by the second phase of SSA higherorder self-association (Fig. 5a-d - blue). Within this concentration range there is still an increasing concentration of SSA at the interface, which will result in a decrease in surface tension, however there is also an increase in higher-order
Table 4 Summary of CMC values calculated from OD and surface tension measurements obtained using the plate reader (described herein) or pendent drop methodology respectively for an $\mathrm{EtOH} / \mathrm{H}_{2} \mathrm{O}$ $(1: 19 \mathrm{v} / \mathrm{v})$ solution. ${ }^{20}$ Here $\mathrm{rt}=$ room temperature (approx. $\left.291 \mathrm{~K}-298 \mathrm{~K}\right)$

\begin{tabular}{|c|c|c|c|c|}
\hline \multirow[b]{3}{*}{ SSA } & \multicolumn{4}{|c|}{ CMC (mM) values } \\
\hline & \multicolumn{3}{|c|}{ OD well scan method } & \multirow{2}{*}{$\begin{array}{l}\text { Pendant drop method } \\
\text { rt }\end{array}$} \\
\hline & $298 \mathrm{~K}$ & $308 \mathrm{~K}$ & $318 \mathrm{~K}$ & \\
\hline 1 & 1.51 & 1.49 & 1.37 & 1.92 \\
\hline 2 & 0.41 & 0.46 & 0.48 & 0.50 (ref. 31) \\
\hline 3 & 8.91 & 8.69 & 7.99 & 9.54 (ref. 31) \\
\hline 4 & 14.86 & 19.64 & 14.42 & 16.20 \\
\hline
\end{tabular}

structures within the sample bulk, as identified by the increasing OD values within the central 'control' (green) region of the well. Therefore, within a SSAs phase two concentration range the samples of increasing SSA concentration will still report a change in surface tension, while a greater proportion of higher-order aggregated structures are able to exist in the sample bulk or 'control' region. The larger this concentration range, the greater the difference is likely to be between the two comparative CMC values reported by these methodologies. Finally, when comparing the CMC values calculated for SSAs 1-4 we observe a general decrease in CMC with increasing temperature for SSAs 1, 3 and 4. Interestingly, for SSA 2 this trend is reversed, however, the differences here are small and therefore may not be significant.

\section{Conclusions}

We have estimated the $\mathrm{CMC}$ for $\mathbf{1 - 4}$ in an $\mathrm{EtOH} / \mathrm{H}_{2} \mathrm{O}(1: 19 \mathrm{v} / \mathrm{v})$ solvent system through both the use of standard pendant drop methodology and the development of our own OD based methodology. This OD methodology not only increases experimental throughput, but also, we believe, may enable the definition of three phases of higher-order SSA amphiphile selfassociation ((i) primary amphiphile aggregation at the solution interface; (ii) increasing aggregate formation at the interface and within the sample bulk; (iii) further aggregation events primarily occurring within the solution bulk), while allowing environmental control of parameters such as temperature. In addition, unlike other traditional methodologies, this novel assay can be fully automated using instrument injector setups and standard operating procedure (SOP) files, reducing manual errors and personnel time.

Finally, we have shown that in DMSO- $d_{6}$ solutions, the anionic constituent of 2-4 self-associates, through hydrogen bond formation to form lower-order species, e.g. dimers. However, 1 was not shown to form these self-associated structures, which we hypothesise to be the result of competitive intramolecular hydrogen bonding events. In an $\mathrm{EtOH} / \mathrm{H}_{2} \mathrm{O}$ or $\mathrm{D}_{2} \mathrm{O}(1: 19 \mathrm{v} / \mathrm{v})$ solution, all SSAs were shown to form higherorder self-associated species however, these species are thought to be present in much lower quantities with 3 in com- 
parison to 1,2 and 4 , which we attribute to the presence of competitive intramolecular hydrogen bonding events within the SSAs aromatic substituent.

\section{Author contributions}

Andzelika Rutkauskaite: Investigation; validation; writing original draft. Kira L. F. Hilton: Investigation; validation; writing - original draft. Giacomo Picci: Investigation; validation. Lisa J. White: Investigation; writing - original draft. Lorraine Croucher: Validation; writing - original draft; Claudia Caltagirone: Funding acquisition; project administration; supervision; writing - review \& editing. Jennifer R. Hiscock: Conceptualization; funding acquisition; project administration; supervision; writing - review \& editing.

\section{Conflicts of interest}

There are no conflicts to declare.

\section{Acknowledgements}

Firstly, we would like to acknowledge the support Kevin Howland (University of Kent) for his mass spectrometry support and Faith Taylor for her support with some preliminary data collection. JH and LW would like to thank the UKRI for JH's Future Leaders Fellowship (MR/T020415/1). AR and $\mathrm{KH}$ would like to thank the University of Kent for funding. Financial support from MIUR (PRIN 2017 project 2017EKCS35), Università degli Studi di Cagliari (FIR 2016-2019), Fondazione di Sardegna (FdS Progetti Biennali di Ateneo, annualità 2020) is gratefully acknowledged by CC and GP.

\section{Notes and references}

1 D. Lombardo, M. A. Kiselev, S. Magazù and P. Calandra, Adv. Condens. Matter Phys., 2015, 151683.

2 D. F. Evans and H. Wennerstrom, The Colloidal Domian where physics, chemistry, and biology meet, VCH Publishers, New York, 1996, pp. 1-515.

3 B. Parshad, S. Prasad, S. Bhatia, A. Mittal, Y. Pan, P. K. Mishra, S. K. Sharma and L. Fruk, RSC Adv., 2020, 10, 42098-42115.

4 D. B. Amabilino, D. K. Smith and J. W. Steed, Chem. Soc. Rev., 2017, 46, 2404-2420.

5 E. R. Draper and D. J. Adams, Chem, 2017, 3, 390-410.

6 A. Pinazo, M. A. Manresa, A. M. Marques, M. Bustelo, M. J. Espuny and L. Pérez, Adv. Colloid Interface Sci., 2016, 228, 17-39.

7 H. Gong, M. Liao, X. Hu, K. Fa, S. Phanphak, D. Ciumac, P. Hollowell, K. Shen, L. A. Clifton, M. Campana,
J. R. P. Webster, G. Fragneto, T. A. Waigh, A. J. Mcbain and J. R. Lu, ACS Appl. Mater. Interfaces, 2020, 12, 44420-44432.

8 C. Zhou and Y. Wang, Curr. Opin. Colloid Interface Sci., 2020, 45, 28-43.

9 G. Dey, R. Bharti, R. Sen and M. Mandal, Drug Discovery Today, 2015, 20, 136-146.

10 C. Chen, Y. Chen, C. Yang, P. Zeng, H. Xu, F. Pan and J. R. Lu, ACS Appl. Mater. Interfaces, 2015, 7, 17346-17355.

11 S. Harrisson, J. Nicolas, A. Maksimenko, D. T. Bui, J. Mougin and P. Couvreur, Angew. Chem., Int. Ed., 2013, 52, 1678-1682.

12 L. Djordjevic, M. Primorac, M. Stupar and D. Krajisnik, Int. J. Pharm., 2004, 271, 11-19.

$13 \mathrm{X} . \mathrm{Hu}, \mathrm{J} . \mathrm{Hu}, \mathrm{J}$. Tian, Z. Ge, G. Zhang, K. Luo and S. Liu, J. Am. Chem. Soc., 2013, 135, 17617-17629.

14 M. P. Krafft, Adv. Drug Delivery Rev., 2001, 47, 209-228.

15 G. Shi, Y. Shen, P. Mu, Q. Wang, Y. Yang, S. Ma and J. Li, Green Chem., 2020, 22, 1345-1352.

16 I. M. Banat, R. S. Makkar and S. S. Cameotra, Appl. Microbiol. Biotechnol., 2000, 53, 495-508.

17 Y. Li, X. He, X. Cao, G. Zhao, X. Tian and X. Cui, J. Colloid Interface Sci., 2007, 307, 215-220.

18 F. K. G. Santos, E. L. B. Neto, M. C. P. A. Moura, T. N. C. Dantas and A. A. D. Neto, Colloids Surf., A, 2009, 333, 156-162.

19 Á. Piñeiro, X. Banquy, S. Pérez-Casas, E. Tovar, A. García, A. Villa, A. Amigo, A. E. Mark and M. Costas, J. Phys. Chem. B, 2007, 111, 4383-4392.

20 J. D. Berry, M. J. Neeson, R. R. Dagastine, D. Y. C. Chan and R. F. Tabor, J. Colloid Interface Sci., 2015, 454, 226-237.

21 F. M. Menger, A. L. Galloway and M. E. Chlebowski, Langmuir, 2005, 21, 9010-9012.

22 P. Goon, C. Manohar and V. V. Kumar, J. Colloid Interface Sci., 1997, 189, 177-180.

23 M. Pérez-Rodríguez, G. Prieto, C. Rega, L. M. Varela, F. Sarmiento and V. Mosquera, Langmuir, 1998, 14, 4422-4426.

24 A. Fluksman and O. Benny, Anal. Methods, 2019, 11, 38103818.

25 J. K. Salem, I. M. El-Nahhal and S. F. Salama, Chem. Phys. Lett., 2019, 730, 445-450.

26 T. Jumpertz, B. Tschapek, N. Infed, S. H. J. Smits, R. Ernst and L. Schmitt, Anal. Biochem., 2011, 408, 64-70.

27 M. M. Mabrouk, N. A. Hamed and F. R. Mansour, Appl. Spectrosc. Rev., 2021, 1-29.

28 Ö. Topel, B. A. Çakir, L. Budama and N. Hoda, J. Mol. Liq., 2013, 177, 40-43.

29 E. Ruckenstein and R. Nagarajan, J. Phys. Chem., 1975, 79, 2622-2626.

30 D. E. Kile and C. T. Chiou, Environ. Sci. Technol., 1989, 23, 832-838.

31 L. J. White, N. J. Wells, L. R. Blackholly, H. J. Shepherd, B. Wilson, G. P. Bustone, T. J. Runacres and J. R. Hiscock, Chem. Sci., 2017, 8, 7620-7630.

32 L. J. White, S. N. Tyuleva, B. Wilson, H. J. Shepherd, K. K. L. Ng, S. J. Holder, E. R. Clark and J. R. Hiscock, Chem. - Eur. J., 2018, 24, 7761-7773. 
33 S. F. Burlatsky, V. V. Atrazhev, D. V. Dmitriev, V. I. Sultanov, E. N. Timokhina, E. A. Ugolkova, S. Tulyani and A. Vincitore, J. Colloid Interface Sci., 2013, 393, 151-160.

34 N. Wu, J. Dai and F. J. Micale, J. Colloid Interface Sci., 1999, 215, 258-269.

35 A. F. Belhaj, K. A. Elraies, M. S. Alnarabiji, J. A. B. M. Shuhli, S. M. Mahmood and L. W. Ern, Energies, 2019, 12, 2319.

36 S. K. Hait and S. P. Moulik, J. Surfactants Deterg., 2001, 4, 303-309.

37 E. Mohajeri and G. D. Noudeh, E-J. Chem., 2012, 9, 22682274.

38 K. G. Furton and A. Norelus, J. Chem. Educ., 1993, 70, 254257.

39 M. S. Alam, A. Z. Naqvi and K. Din, J. Chem. Eng. Data, 2007, 52, 1326-1331.

40 L. Cai, M. Gochin and K. Liu, Chem. Commun., 2011, 47, 5527-5529.

41 T. L. Gumbs, L. J. White, N. J. Wells, H. J. Shepherd and J. R. Hiscock, Supramol. Chem., 2018, 30, 42-51.

42 K. K. L. Ng, M. Dimitrovski, J. E. Boles, R. J. Ellaby, L. J. White and J. R. Hiscock, Supramol. Chem., 2020, 32, 414-424.

43 J. R. Hiscock, G. P. Bustone, B. Wilson, K. E. Belsey and L. R. Blackholly, Soft Matter, 2016, 12, 4221-4228.

44 L. R. Blackholly, H. J. Shepherd and J. R. Hiscock, CrystEngComm, 2016, 18, 7021-7028.

45 S. N. Tyuleva, N. Allen, L. J. White, A. Pépés, H. J. Shepherd, P. J. Saines, R. J. Ellaby, D. P. Mulvihill and J. R. Hiscock, Chem. Commun., 2018, 55, 95-98.
46 N. Allen, L. J. White, J. E. Boles, G. T. Williams, D. F. Chu, R. J. Ellaby, H. J. Shepherd, K. K. L. Ng, L. R. Blackholly, B. Wilson, D. P. Mulvihill and J. R. Hiscock, ChemMedChem, 2020, 15, 2193-2205.

47 L. J. White, J. E. Boles, N. Allen, L. S. Alesbrook, J. M. Sutton, C. K. Hind, K. L. F. Hilton, L. R. Blackholly, R. J. Ellaby, G. T. Williams, D. P. Mulvihill and J. R. Hiscock, J. Mater. Chem. B, 2020, 8, 4694-4700.

48 J. E. Boles, R. J. Ellaby, H. J. Shepherd and J. R. Hiscock, RSC Adv. , 2021, 11, 9550-9556.

49 N. O. Dora, E. Blackburn, J. E. Boles, G. T. Williams, L. J. White, S. E. G. Turner, J. D. Hothersall, T. Askwith, J. A. Doolan, D. P. Mulvihill, M. D. Garrett and J. R. Hiscock, RSC Adv., 2021, 11, 14213-14217.

50 K. K. L. Ng, R. Devlia, N. L. Foss, L. S. Alesbrook, J. R. Hiscock and A. T. Murray, Chem. Commun., 2020, 56, 11815-11818.

51 L. J. White, J. E. Boles, K. L. F. Hilton, R. J. Ellaby and J. R. Hiscock, Molecules, 2020, 25, 4126.

52 Bindfit v0.5, http://app.supramolecular.org/bindfit/ (accessed 20/10/2021).

53 B. Kuhn, P. Mohr and M. Stahl, J. Med. Chem., 2010, 53, 2601-2611.

54 L. J. White, C. Wark, L. Croucher, E. R. Draper and J. R. Hiscock, Chem. Commun., 2020, 56, 9557-9560.

55 G. M. Sheldrick, Acta Crystallogr., Sect. A: Found. Adv., 2015, 3-8. 56 G. M. Sheldrick, Acta Crystallogr., Sect. C: Struct. Chem., 2015, 71, 3-8.

57 O. V. Dolomanov, L. J. Bourhis, R. J. Gildea, J. A. K. Howard and H. Puschmann, J. Appl. Crystallogr., 2009, 42, 339-341. 